\title{
Non-Markovian dynamics in plasmon-induced spontaneous emission interference
}

\author{
I. Thanopulos ${ }^{1}$ V. Yannopapas ${ }^{2}$ 团 and E. Paspalakis ${ }^{2}$ \\ 1 Department of Optics and Optometry, T.E.I. of Western Greece, Aigio 251 00, Greece \\ 2 Department of Physics, National Technical University of Athens, Athens 157 80, Greece and \\ ${ }^{3}$ Department of Materials Science, School of Natural Sciences, University of Patras, Patras 265 04, Greece
}

\begin{abstract}
We investigate theoretically the non-Markovian dynamics of a degenerate $\mathrm{V}$-type quantum emitter in the vicinity of a metallic nanosphere, a system that exhibits quantum interference in spontaneous emission due to the anisotropic Purcell effect. We calculate numerically the electromagnetic Green's tensor and employ the effective modes differential equation method for calculating the quantum dynamics of the emitter population, with respect to the resonance frequency and the initial state of the emitter, as well as its distance from the nanosphere. We find that the emitter population evolution varies between a gradually total decay and a partial decay combined with oscillatory population dynamics, depending strongly on the specific values of the above three parameters. Under strong coupling conditions, coherent population trapping can be observed in this system. We compare our exact results with results when the flat continuum approximation for the modified by the metallic nanosphere vacuum is applied. We conclude that the flat continuum approximation is an excellent approximation only when the spectral density of the system under study is characterized by non-overlapping plasmonic resonances.
\end{abstract}

PACS numbers: 42.50.Nn, 42.50.Ct, 73.20.Mf, 78.67.Bf

\section{INTRODUCTION}

Metallic nanoparticles (MNPs) can confine light inside deep subwavelength volumes in electromagnetic (EM) modes, which are called localized surface plasmons (LSPs). This strong field localization leads to an enhancement of the light-matter interaction in the vicinity of the MNP, featuring some very interesting physics in a broad range of contexts such as optical antennas 1.2 , surface-enhanced Raman scattering ${ }^{3}$, photovoltaics ${ }^{4}$, energy transfer in light-harvesting ${ }^{\frac{5}{}}$ and biosensing ${ }^{6}$.

The presence of LSPs in metal-dielectric interfaces strongly modifies the density of the EM modes in the surroundings, leading to strong modification of the lifetime of quantum emitters (QE) placed close to the MNP. Moreover, when the distance between the QE and the MNP surface is very small, theory predicts that coherent energy exchange between the $\mathrm{QE}$ and the modified EM modes accompanied with non-Markovian dynamics may take place ${ }^{7-12}$. The above phenomena have been demonstrated mostly experimentally in the perturbative (weak-coupling) regime wherein quantum dynamics is irreversible $\underline{13-19}$. Nevertheless, recently strong coupling at room temperature between an organic molecule and a plasmonic nanocavity has been demonstrated experimentally ${ }^{20}$, followed by a detailed theoretical analysis 21 .

In this work we study theoretically the population dynamics of a V-type QE coupled electromagnetically to a MNP. The spontaneous emission of such a QE may exhibit interference effects due the fact that the MNP affects differently the EM modes along mutually perpendicular directions, known as the anisotropic Purcell effect ${ }^{22,23}$. This phenomenon has been studied in various photonic structures, including periodic dielectrics 24 , nega- tive refractive index metamaterials $\frac{25-27}{2}$, metasurfaces $\frac{28}{}$, hyperbolic metamaterials 29 , as well as plasmonic nanostructures $30-32$.

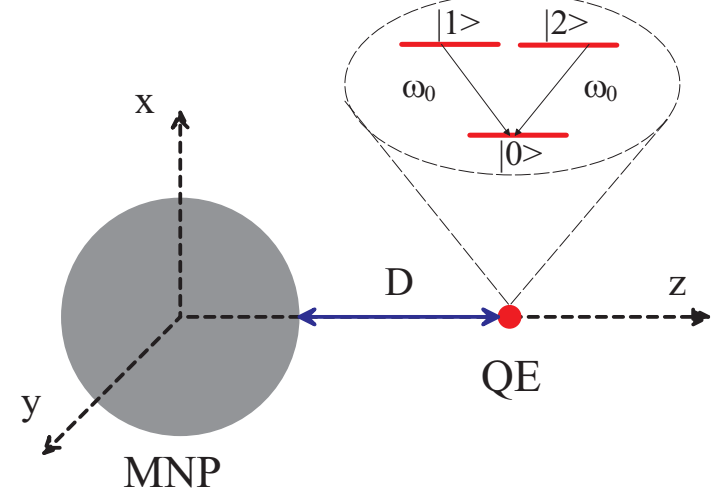

FIG. 1: (color online) The configuration of the degenerate $\mathrm{V}$ type QE with resonance frequency $\omega_{0}$, placed at a distance $D$ from the surface of a spherical MNP.

Here, in contrast to previous work ${ }^{22}-32$, the interaction of the QE with the MNP is not restricted to the weakcoupling regime. We use a quantum-mechanical formalism for the EM field excitations ${ }^{9}-11$, which goes beyond the Markov approximation and incorporates the exact population dynamics by employing the effective mode differential equation (EMDE) method ${ }^{33}$. The anisotropic enhancement of the spontaneous decay rates of a $\mathrm{QE}$ in the proximity of a MNP is calculated using an EM Green's tensor technique ${ }^{34}$. The purpose of the present work is to study in detail the crossover regime in which 
the QE population dynamics changes from irreversible to reversible for a system shown in Fig. 1 manifested by the emergence of non-decaying oscillations in the evolution of the QE population. Such oscillations signify the coherent exchange of energy between the QE and modified reservoir of EM modes due to the nearby MNP.

This paper is organized as follows: In Section [I] we present our methodology for calculating the spontaneous emission dynamics of a QE near a MNP. We then present and discuss the results for a V-type system in Section $\amalg$. We also present relevant results for a two-level system in Section IV Finally, we conclude our findings in Section V]

\section{CALCULATION OF THE SPONTANEOUS EMISSION DYNAMICS OF A $V$-TYPE QE NEAR A MNP}

We consider a degenerate $\mathrm{V}$-type $\mathrm{QE}$ at distance $D$ from the surface of a spherical MNP, as shown in Fig. 1. The origin of the coordinate system coincides with the center of the sphere and the QE lies on the $\mathrm{z}$ axis of the coordinate system. The Hamiltonian of the system 9,11 , is given by (we use $\hbar=1$ throughout this work)

$$
\begin{aligned}
H & =\sum_{i=1,2} \omega_{0} \hat{\sigma}_{i i}+\int d \vec{r} \int_{0}^{\infty} d \omega \omega \hat{\mathbf{f}}^{\dagger}(\vec{r}, \omega) \cdot \hat{\mathbf{f}}(\vec{r}, \omega) \\
& -\sum_{i=1,2}\left[\hat{\sigma}_{i 0} \int_{0}^{\infty} d \omega \vec{\mu}_{i 0} \cdot \hat{\mathbf{E}}(\vec{r}, \omega)+H . c .\right] .
\end{aligned}
$$

In Eq. (11), $\hat{\mathbf{f}}(\vec{r}, \omega), \hat{\mathbf{f}}^{\dagger}(\vec{r}, \omega)$ stand for the bosonic vector field operators for the elementary excitations of the system, $\hat{\sigma}_{i j}$ denotes the Pauli operator, $\vec{\mu}_{10}=\mu \hat{\varepsilon}_{-}$and $\vec{\mu}_{20}=\mu \hat{\varepsilon}_{+}$are the dipole moments of the $\mathrm{QE}$, where $\hat{\varepsilon}_{ \pm} \equiv\left(\hat{\varepsilon}_{z} \pm i \hat{\varepsilon}_{x(y)}\right) / \sqrt{2}$ describe the right-rotating $\left(\hat{\varepsilon}_{+}\right)$ and left-rotating $\left(\hat{\varepsilon}_{-}\right)$unit vectors ( $\mu$ is taken to be real). Also, $\omega_{0}$ stands for the resonance frequency between the two degenerate upper levels and the lower level of the V-type QE, with the energy of the lower level taken as zero.

The electric field vector operator $\hat{\mathbf{E}}(\vec{r}, \omega)$ is given by

$$
\hat{\mathbf{E}}(\vec{r}, \omega)=\frac{i \omega^{2}}{\sqrt{\pi \epsilon_{0}} c^{2}} \int d \vec{s} \sqrt{\operatorname{Im}[\epsilon(\vec{s}, \omega)]} \hat{\mathbf{G}}(\vec{r}, \vec{s}, \omega) \cdot \hat{\mathbf{f}}(\vec{s}, \omega),
$$

with $\hat{\mathbf{G}}(\vec{r}, \vec{s}, \omega)$ being the dyadic EM Green's tensor defined as

$$
\nabla \times \nabla \times \hat{\mathbf{G}}(\vec{r}, \vec{s}, \omega)-\frac{\epsilon(\vec{r}, \omega) \omega^{2}}{c^{2}} \hat{\mathbf{G}}(\vec{r}, \vec{s}, \omega)=\hat{\mathbf{I}} \delta(\vec{r}-\vec{s}) .
$$

In Eqs. (2) and (3), $\hat{\mathbf{I}}$ is the unit dyad (unit tensor), $\epsilon(\vec{r}, \omega)$ is the spatially and frequency dependent complex dielectric function of the MNP, and $c$ is the speed of light in the vacuum.
The state of the system is given by

$$
\begin{aligned}
|\Psi(t)\rangle= & c_{1}(t) e^{-i \omega_{0} t}\left|1 ; 0_{\omega}\right\rangle+c_{2}(t) e^{-i \omega_{0} t}\left|2 ; 0_{\omega}\right\rangle+ \\
& \int d \vec{r} \int d \omega C(\vec{r}, \omega, t) e^{-i \omega t}\left|0 ; 1_{\vec{r}, \omega}\right\rangle,
\end{aligned}
$$

where $\left|0 ; 1_{\vec{r}, \omega}\right\rangle \equiv \hat{f}_{\lambda}^{\dagger}(\vec{r}, \omega)\left|0 ; 0_{\omega}\right\rangle$. Here, $|n ; a\rangle=|n\rangle \otimes$ $|a\rangle$, where $|n\rangle(n=0,1,2)$ denotes the quantum states of the V-type system (see Fig. 1) and $|a\rangle$ denotes the photonic states (states of the modified EM vacuum), with $\left|0_{\omega}\right\rangle$ meaning zero and $\left|1_{\vec{r}, \omega}\right\rangle$ one photon states. The application of the time-dependent Schrödinger equation $i|\dot{\Psi}(t)\rangle=H|\Psi(t)\rangle$ yields a set of differential equations that can be formally integrated, resulting in two integrodifferential equations for $c_{1}(t)$ and $c_{2}(t)$, given by

$$
\dot{c}_{1}(t)=i \int_{0}^{t} d t^{\prime}\left(K^{11}\left(t-t^{\prime}\right) c_{1}\left(t^{\prime}\right)+K^{12}\left(t-t^{\prime}\right) c_{2}\left(t^{\prime}\right)\right),
$$

$$
\dot{c}_{2}(t)=i \int_{0}^{t} d t^{\prime}\left(K^{21}\left(t-t^{\prime}\right) c_{1}\left(t^{\prime}\right)+K^{22}\left(t-t^{\prime}\right) c_{2}\left(t^{\prime}\right)\right),
$$

where

$$
\begin{aligned}
K^{i j}(\tau) & =i \frac{e^{i \omega_{0} \tau}}{\pi} \int_{0}^{\infty} \mathcal{G}^{i j}(\omega) e^{-i \omega \tau} d \omega \\
\mathcal{G}^{i j}(\omega) & \equiv \frac{\omega^{2}}{\epsilon_{0} c^{2}}\left[\vec{\mu}_{i 0}^{\dagger} \cdot \operatorname{Im}\left[\hat{\mathbf{G}}\left(\vec{r}_{0}, \vec{r}_{0}, \omega\right)\right] \cdot \vec{\mu}_{j 0}\right],
\end{aligned}
$$

with $\tau \equiv t-t^{\prime}$ and $i, j=1,2$.

In general, the MNP affects differently the EM modes along the tangential $(\|)$ and the radial $(\perp)$ directions, corresponding to a $\mathrm{QE}$ with a transition dipole oriented parallel and perpendicular to the surface of the MNP, respectively. In our case, the radial direction coincides with the $z$ axis, and the tangential direction is parallel to the $x$ and $y$ axes, according to Fig. 1. In such a case, we have $(i, j=1,2)$

$$
K^{i i}(\tau)=K^{+}(\tau)=i e^{i \omega_{0} \tau} \int_{0}^{\infty} J^{+}(\omega) e^{-i \omega \tau} d \omega,
$$

$$
K^{i j}(\tau)=K^{-}(\tau)=i e^{i \omega_{0} \tau} \int_{0}^{\infty} J^{-}(\omega) e^{-i \omega \tau} d \omega, i \neq j
$$

with

$$
\begin{aligned}
J^{ \pm}(\omega) & =\frac{1}{2 \pi} \frac{\omega^{2} \mu^{2}}{\epsilon_{0} c^{2}} \operatorname{Im}\left[\hat{\mathbf{G}}_{\perp}\left(\vec{r}_{0}, \vec{r}_{0}, \omega\right) \pm \hat{\mathbf{G}}_{\|}\left(\vec{r}_{0}, \vec{r}_{0}, \omega\right)\right] \\
& \equiv J^{\perp}(\omega) \pm J^{\|}(\omega),
\end{aligned}
$$

where $\hat{\mathbf{G}}_{\perp} \equiv \hat{\mathbf{G}}_{z z}$ and $\hat{\mathbf{G}}_{\|} \equiv \hat{\mathbf{G}}_{x x(y y)}$. The decay rate $\Gamma(\omega)$ in the presence of a MNP is given by ${ }^{35}$

$$
\Gamma^{k}(\omega)=\frac{2 \mu^{2} \omega^{2}}{\epsilon_{0} c^{2}} \operatorname{Im}\left[\hat{\mathbf{G}}_{k}\left(\vec{r}_{0}, \vec{r}_{0}, \omega\right)\right], \quad k=\perp, \|,
$$


and we thus obtain

$$
J^{k}(\omega)=\frac{1}{2 \pi} \frac{\Gamma^{k}(\omega)}{2}=\frac{1}{2 \pi} \frac{\lambda^{k}(\omega, D) \Gamma_{0}(\omega)}{2},
$$

with $\Gamma_{0}(\omega)$ being the decay rate of the QE in freespace, and $\lambda^{k}(\omega, D)$ is the directional $(k=\perp, \|)$ enhancement factor of the free-space decay rate due to the presence of the MNP at distance $D$ from the QE. Due to the anisotropy, the enhancement factors $\lambda^{\|}(\omega, D)$ and $\lambda^{\perp}(\omega, D)$ are not equal, in general. We further write

$$
\Gamma_{0}(\omega)=\frac{\omega_{0}^{3} \mu^{2}}{3 \pi \epsilon_{0} c^{3}}\left(\frac{\omega}{\omega_{0}}\right)^{3} \equiv \Gamma_{0}\left(\omega_{0}\right)\left(\frac{\omega}{\omega_{0}}\right)^{3},
$$

since in free-space $\operatorname{Im}\left[\hat{\mathbf{G}}_{0}\left(\vec{r}_{0}, \vec{r}_{0}, \omega\right)\right]=\frac{\omega}{6 \pi c} \hat{\mathbf{I}}$ is valid ${ }^{12,35}$, with $\hat{\mathbf{G}}_{0}\left(\vec{r}_{0}, \vec{r}_{0}, \omega\right)$ being the free-space Green's tensor. We now obtain

$$
\begin{aligned}
K^{ \pm}(\tau) & =i \int_{0}^{\infty} J^{ \pm}(\omega) e^{-i\left(\omega-\omega_{0}\right) \tau} d \omega \\
J^{ \pm}(\omega) & \equiv \frac{\Gamma_{0}\left(\omega_{0}\right)}{2 \pi} \frac{\lambda^{\perp}(\omega, D) \pm \lambda^{\|}(\omega, D)}{2}\left(\frac{\omega}{\omega_{0}}\right)^{3} \\
& \equiv J^{r a d}(\omega) \pm J^{\tan }(\omega),
\end{aligned}
$$

with $\Gamma_{0}\left(\omega_{0}\right)=1 / \tau_{0}$, and $\tau_{0}$ standing for the freespace decay time of the QE. Taking into account that $\exp \left(-i\left(\omega-\omega_{0}\right) \tau\right)$ contributes mainly around $\omega_{0}$, we can write $\left(\frac{\omega}{\omega_{0}}\right)^{3} \approx 1$, introducing thus the flat continuum approximation (FCA), and obtaining

$$
\begin{aligned}
K^{ \pm}(\tau) & \approx K_{F C A}^{ \pm}(\tau) \\
K_{F C A}^{ \pm} & =i \int_{0}^{\infty} J_{F C A}^{ \pm}(\omega) e^{-i\left(\omega-\omega_{0}\right) \tau} d \omega \\
J_{F C A}^{ \pm}(\omega) & \equiv \frac{\Gamma_{0}\left(\omega_{0}\right)}{2 \pi} \frac{\lambda^{\perp}(\omega, D) \pm \lambda^{\|}(\omega, D)}{2} \\
& \equiv J_{F C A}^{r a d}(\omega) \pm J_{F C A}^{\tan }(\omega) .
\end{aligned}
$$

We can now expand (to any given accuracy) $K^{ \pm}(\tau)$ as a sum of $M$ exponential terms at effective mode frequencies $\omega_{i}(i=1, \ldots, M)^{33}$,

$$
K^{ \pm}(\tau)=i e^{i \omega_{0} \tau} \sum_{i=1}^{M} W_{i}^{ \pm} e^{-i \omega_{i} \tau}
$$

Then, the equations for the two upper states of the QE are given by

$$
\dot{c}_{\nu}(t)=i \sum_{i=1}^{M} e^{i\left(\omega_{0}-\omega_{i}\right) t} \mathcal{J}_{i}^{\nu}(t), \nu=1,2
$$

where we have introduced new variables given by

$$
\mathcal{J}_{i}^{1}(t)=i \int_{0}^{t}\left(W_{i}^{+} c_{1}\left(t^{\prime}\right)+W_{i}^{-} c_{2}\left(t^{\prime}\right)\right) e^{-i\left(\omega_{0}-\omega_{i}\right) t^{\prime}} d t^{\prime}
$$

$$
\mathcal{J}_{i}^{2}(t)=i \int_{0}^{t}\left(W_{i}^{-} c_{1}\left(t^{\prime}\right)+W_{i}^{+} c_{2}\left(t^{\prime}\right)\right) e^{-i\left(\omega_{0}-\omega_{i}\right) t^{\prime}} d t^{\prime}
$$

for which holds

$$
\begin{aligned}
\dot{\mathcal{J}}_{i}^{1}(t) & =i\left(W_{i}^{+} c_{1}(t)+W_{i}^{-} c_{2}(t)\right) e^{-i\left(\omega_{0}-\omega_{i}\right) t}, \\
\dot{\mathcal{J}}_{i}^{2}(t) & =i\left(W_{i}^{-} c_{1}(t)+W_{i}^{+} c_{2}(t)\right) e^{-i\left(\omega_{0}-\omega_{i}\right) t} .
\end{aligned}
$$

In this way, we have transformed the two integrodifferential equations, Eqs. (5), (6), into a set of $2(M+1)$ effective modes (ordinary) differential equations (EMDE) with constant coefficients, given by Eqs. (21), (24) and (25). Denoting $\tilde{C}_{\nu}(t) \equiv \exp \left(-i \omega_{0} t\right) c_{\nu}(t)$ and $\tilde{\mathcal{J}}_{i}^{\nu}(t) \equiv$ $\exp \left(-i \omega_{i} t\right) \mathcal{J}_{i}^{\nu}(t)$, with $\nu=1,2$ and $i=1, \ldots, M$, we obtain Eqs. (21), (24), and (25) in a matrix form

$$
\dot{\tilde{\mathbf{C}}}(t)=i \tilde{\mathbf{H}} \cdot \tilde{\mathbf{C}}(t),
$$

where $\tilde{\mathbf{C}}(t)=\left(\tilde{C}_{1}(t), \tilde{C}_{2}(t), \tilde{\mathcal{J}}_{1}^{1}(t), \ldots, \tilde{\mathcal{J}}_{M}^{1}(t), \tilde{\mathcal{J}}_{1}^{2}(t), \ldots, \tilde{\mathcal{J}}_{M}^{2}(t)\right)^{\top}$ $\equiv \quad\left(\tilde{C}_{1}(t), \tilde{C}_{2}(t), \tilde{\mathcal{J}}^{(1)}(t), \ldots, \tilde{\mathcal{J}}^{(2 M)}(t)\right)^{\top}, \quad$ with all $\tilde{\mathcal{J}}^{(i)}(0)=0$. Here the superscript $\mathrm{T}$ is the transpose operation and $\tilde{\mathbf{H}}$ is a time-independent complex matrix, given by

$$
\tilde{\mathbf{H}}=\left(\begin{array}{ll}
\mathcal{S} & \mathcal{Y} \\
\mathcal{Z} & \mathcal{R}
\end{array}\right), \quad \mathcal{Z}=\left(\begin{array}{l}
\mathrm{W}^{(1)} \\
\ldots \\
\mathrm{W}^{(2 M)}
\end{array}\right), \mathcal{Y}=\left(\begin{array}{l}
\Omega^{(1)} \\
\Omega^{(2)}
\end{array}\right)
$$

In Eq. (27), we have $\mathcal{S}_{k, k^{\prime}}=\delta_{k, k^{\prime}} \omega_{0},\left(k, k^{\prime}=1,2\right)$, and $\mathcal{R}_{i, i^{\prime}}=\mathcal{R}_{i+M, i^{\prime}+M}=\delta_{i, i^{\prime}} \omega_{i},\left(i, i^{\prime}=1, \ldots, M\right)$, for the diagonal blocks. Furthermore, the $2 M$ rows of $\mathcal{Z}$ are defined as $\mathrm{W}^{(\eta)}=\left(W_{\eta}^{+}, W_{\eta}^{-}\right)$and $\mathrm{W}^{(M+\eta)}=\left(W_{\eta}^{-}, W_{\eta}^{+}\right)$, for $\eta=1, \ldots, M$. Lastly, the two rows of $\mathcal{Y}$ are defined as $\left[\Omega^{(1)}\right]_{1, m}=\left[\Omega^{(2)}\right]_{2, n}=1$, for $3 \leq m \leq 2+M$ and $3+M \leq n \leq 2(M+1)$, with $\left[\Omega^{(1)}\right]_{1, m}=\left[\Omega^{(2)}\right]_{1, n}=0$, for $m$ and $n$ otherwise. Given these definitions we write the solution of Eq. (26) as,

$$
\tilde{\mathbf{C}}(t)=\sum_{j=1}^{M^{\prime}} \Lambda_{j} e^{i \lambda_{j} t} \tilde{C}_{j}^{0},
$$

where $M^{\prime}=2(M+1)$ is the number of $\lambda_{j}$ eigenvalues and $\boldsymbol{\Lambda}_{j}$ eigenvectors of $\tilde{\mathbf{H}}$. The vector of complex coefficients $\tilde{C}_{j}^{0}$ is given as $\tilde{\mathbf{C}}^{\mathbf{0}}=\mathbf{L}^{-1} \cdot \tilde{\mathbf{C}}(0)$. The columns of the $\mathbf{L} \equiv\left[\boldsymbol{\Lambda}_{1}, \ldots, \boldsymbol{\Lambda}_{M^{\prime}}\right]$ matrix are the eigenvectors of $\tilde{\mathbf{H}}$. We note that since $\tilde{\mathbf{H}}$ is not Hermitian, $\mathbf{L}^{-1} \neq \mathbf{L}^{\dagger}$.

The EM response of the $5 \mathrm{~nm}$ silver sphere used in this study is described by a Drude dielectric function, $\epsilon_{m}(\omega)=\epsilon_{m, \infty}-\omega_{p}^{2} /\left(\omega^{2}+i \omega \gamma\right)$, characterized by its plasma frequency $\omega_{p}=9.176 \mathrm{eV}$, high-frequency component $\epsilon_{m, \infty}=3.718 \mathrm{eV}$, and Ohmic losses $\gamma=0.021$ $\mathrm{eV}$. The radial and tangential enhancement factors of the free-space decay rate of the $\mathrm{QE}$ due to the presence of the MNP as a function of frequency $\omega$ at distance $D$, calculated using an EM Green's tensor technique ${ }^{34}$, are presented in Fig. 2. 

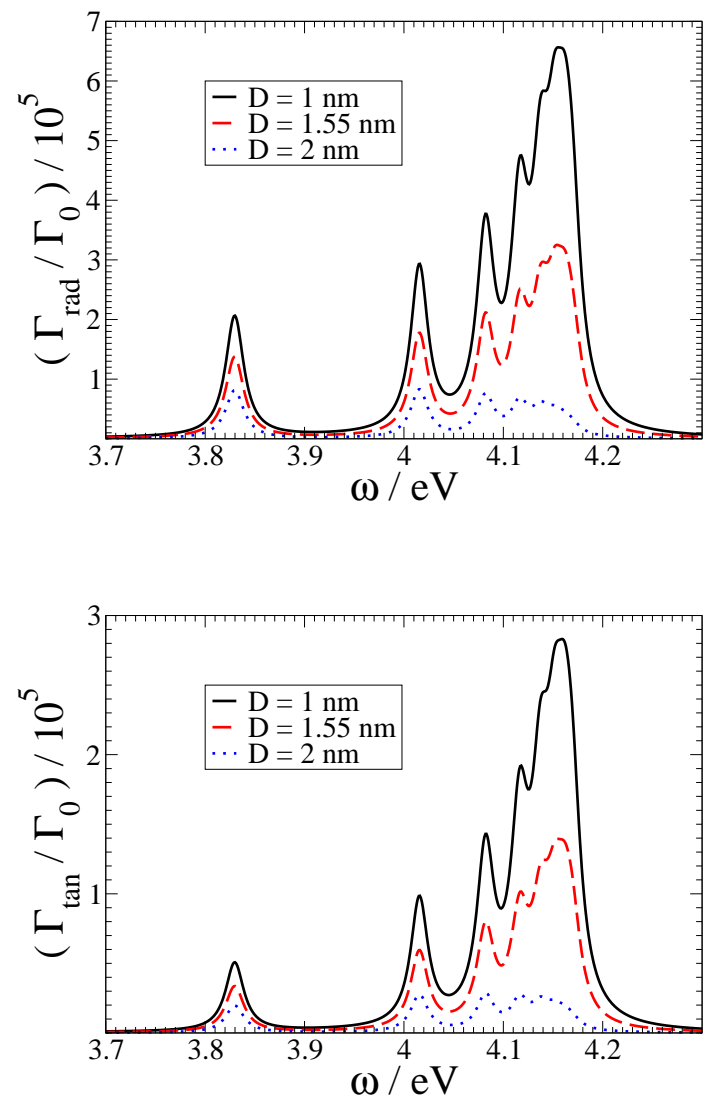

FIG. 2: (color online) Radial $\lambda^{\perp}(\omega, D) \equiv \Gamma_{\text {rad }} / \Gamma_{0}$ (top panel) and tangential $\lambda^{\|}(\omega, D) \equiv \Gamma_{\text {tan }} / \Gamma_{0}$ (bottom panel) enhancement factors as function of the distance $D$ of the QE from the MNP and EM mode frequency $\omega$.

We note that our results below are converged with respect to the number of effective modes $M$ included in the dynamics; we use $M=1001$ in the energy range 3.5-4.5 $\mathrm{eV}$. We also note that our results on $\mathrm{V}$-type and two-level systems presented below are obtained using the $K^{ \pm}(\tau)$ kernel as defined in Eq. (15) unless otherwise stated.

\section{DYNAMICS OF A V-TYPE QE NEAR A MNP}

In this work, we investigate the $\left|c_{1}(t)\right|^{2}$ and $\left|c_{2}(t)\right|^{2}$ time evolution for state-of-the-art QEs with transition frequencies in the optical regime, such as quantum dots (QD) and J-aggregates (J-AGR), with free-space decay time $\tau_{0} \approx 4 \mathrm{~ns}^{36}$ and $\approx 70 \mathrm{ps} \frac{37}{}$, respectively; correspondingly, we often use the terms QD and J-AGR for referring to QEs with such free-space decay times below. In each case, we study the dynamics for different initial states of the QE. Moreover, for each QE, we consider two resonance frequencies, $\omega_{0}=3.84 \mathrm{eV}$ and $\omega_{0}=4.16 \mathrm{eV}$, the frequencies corresponding to (relatively) small and large enhancement factors $\lambda^{\|}\left(\omega_{0}, D\right)$ and $\lambda^{\perp}\left(\omega_{0}, D\right)$, respectively, shown in Fig. 2. We also define the effective decay time of a $\mathrm{QE}$ with resonance frequency $\omega_{0}$ and freespace decay rate $\tau_{0}$ at distance $D$ from the MNP surface as $\tau_{\text {eff }} \equiv\left(\tau_{\text {eff }}^{r a d}+\tau_{\text {eff }}^{\text {tan }}\right) / 2$, given by the average of the corresponding radial $\tau_{\text {eff }}^{r a d} \equiv \tau_{0} / \lambda_{\perp}\left(\omega_{0}, D\right)$ and tangential $\tau_{\text {eff }}^{t a n} \equiv \tau_{0} / \lambda_{\|}\left(\omega_{0}, D\right)$ effective decay times.

\section{A. QD dynamics at $\omega_{0}=3.84 \mathbf{e V}$}
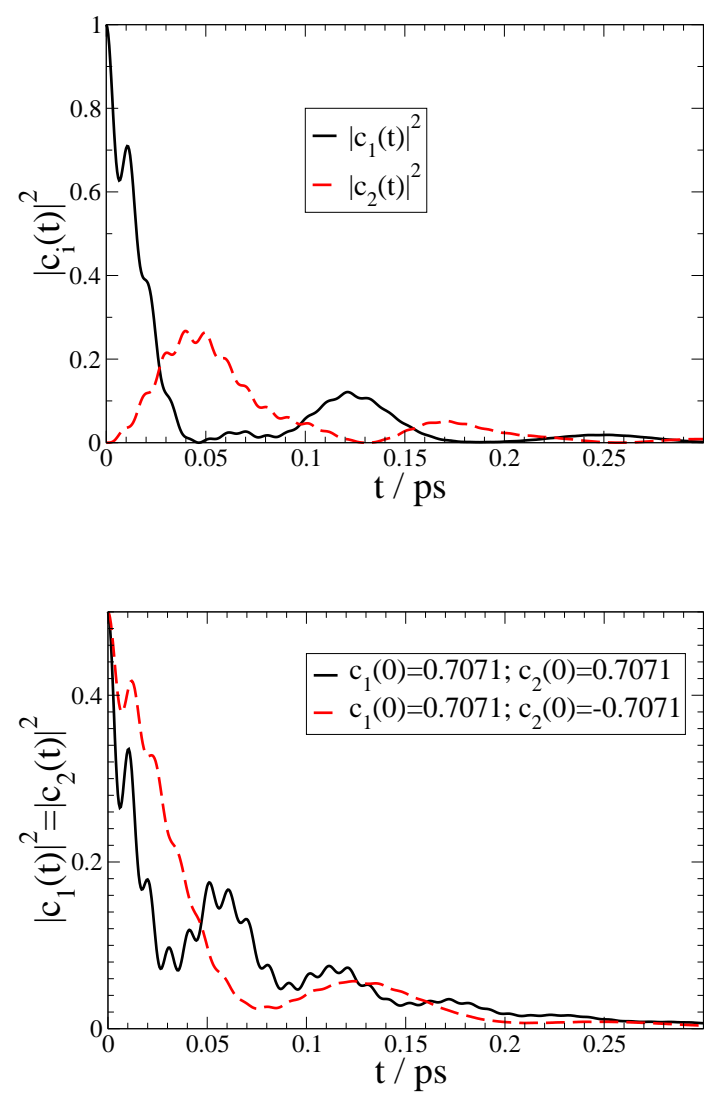

FIG. 3: (color online) Top: Population evolution of $|1\rangle$ (black solid curve) and $|2\rangle$ (red dashed curve) of a QD with $\omega_{0}=$ $3.84 \mathrm{eV}$ at $D=1 \mathrm{~nm}$. Bottom: Population evolution of $|1\rangle$ of the $\mathrm{QD}$ with $\omega_{0}=3.84 \mathrm{eV}$ at $D=1 \mathrm{~nm}$ for the initial states, $|\Psi(0)\rangle=\sqrt{0.5}|1\rangle+\sqrt{0.5}|2\rangle$ (black solid curve) and $|\Psi(0)\rangle=\sqrt{0.5}|1\rangle-\sqrt{0.5}|2\rangle$ (red dashed curve).

The population dynamics of a $\mathrm{QD}$, i.e. a $\mathrm{QE}$ with $\tau_{0}=4 \mathrm{~ns}$, with $\omega_{0}=3.84 \mathrm{eV}$ at $D=1 \mathrm{~nm}$ for different initial states is shown in Fig. 3. In the top panel of this figure, the time-evolution of $\left|c_{1}(t)\right|^{2}$ (black solid curve) and $\left|c_{2}(t)\right|^{2}$ (red dashed curve) is shown for the initial state $|\Psi(0)\rangle=|1\rangle$. We find that the initial population in state $|1\rangle$ decays rapidly into the modified EM modes, 
while there is a partial population transfer from state $|1\rangle$ into state $|2\rangle$ within about $50 \mathrm{fs}$. Partial revivals of the populations in $|1\rangle$ and $|2\rangle$ at $125 \mathrm{fs}$ and $175 \mathrm{fs}$, respectively, are further observed, before all population finally ends up in the modified EM continuum after about $250 \mathrm{fs}$. The effective decay time for a $\mathrm{QE}$ with $\tau_{0}=4 \mathrm{~ns}$, as here, is $\tau_{\text {eff }} \approx 89 \mathrm{fs}\left(\tau_{\text {eff }}^{\text {rad }} \approx 35 \mathrm{fs}\right.$ and $\left.\tau_{\text {eff }}^{\text {tan }} \approx 143 \mathrm{fs}\right)$ considering that $\lambda_{\perp}(3.84,1) \approx 1.13 \cdot 10^{5}$ and $\lambda_{\|}(3.84,1) \approx 2.8 \cdot 10^{4}$ as shown in Fig. 2] The time-evolution of state $|1\rangle$ shown in the top panel of Fig. 3. at early times, is practically a decay, characterised primarily by the decay time $\tau_{\text {eff }}^{r a d}$, instead of $\tau_{e f f}$, indicating that the clearly non-Markovian dynamics are dominated by the radial part (along the $z$ axis) of the modified by the MNP spectral density of the EM modes. Moreover, small oscillations on the population evolution dynamics of $|1\rangle$ and $|2\rangle$, which are discernible also in the top panel of Fig. 3 further point to a rather moderate non-Markovian character. There is also a partial oscillatory exchange of population between state $|1\rangle$ and state $|2\rangle$ via the modified EM modes as indicated by a phase difference, which we define as $\pi$, since, when one is at a (local) maximum the other is at a (local) minimum, and vice versa.

In the bottom panel of Fig. 3 we present the population dynamics for $|1\rangle$ and $|2\rangle$ for the symmetric initial state $|\Psi(0)\rangle=\sqrt{0.5}|1\rangle+\sqrt{0.5}|2\rangle$ (black solid curve) and the antisymmetric initial state $|\Psi(0)\rangle=\sqrt{0.5}|1\rangle-\sqrt{0.5}|2\rangle$ (red dashed curve), denoted by SIS and AIS, respectively. We observe that the population evolution $\left|c_{1}(t)\right|^{2}$, which in case of SIS and AIS equals to $\left|c_{2}(t)\right|^{2}$, decays fast into the modified EM modes for both initial conditions; however, the explicit dynamics depends on the initial state. In case of a SIS, the population decay of $|1\rangle$ is slower than in case of an AIS; in addition, in the latter case, the non-Markovian character of the underlying dynamics is apparently stronger. The differences in the population evolution of state $|1\rangle$ and state $|2\rangle$ in case of a SIS and an AIS clearly indicate that quantum interference effects affect the underlying dynamics, as well as the strength of the non-Markovian character on it. Furthermore, we observe that the population dynamics with a SIS and an AIS, at early times, can be understood as decays, with decay times $\tau_{\text {eff }}^{t a n} \approx 145$ fs and $\tau_{\text {eff }}^{r a d} \approx 35$ fs, respectively; apparently, the dynamics with a SIS is dominated by the tangential Purcell effect and the dynamics with an AIS is dominated by the radial Purcell effect.

\section{B. J-AGR dynamics at $\omega_{0}=3.84 \mathrm{eV}$}

We now focus on the dynamics of a J-AGR, i.e. a QE with $\tau_{0}=70 \mathrm{ps}$, with resonance frequency $\omega_{0}=3.84 \mathrm{eV}$ at $D=2 \mathrm{~nm}$, and corresponding $\tau_{\text {eff }} \approx 4 \mathrm{fs}\left(\tau_{\text {eff }}^{\text {rad }} \approx\right.$ $1.6 \mathrm{fs}$ and $\tau_{\text {eff }}^{\mathrm{tan}} \approx 6.5 \mathrm{fs}$ ), after taking into account that $\lambda_{\perp}(3.84,2) \approx 4.40 \cdot 10^{4}$ and $\lambda_{\|}(3.84,2) \approx 1.07 \cdot 10^{4}$ as shown in Fig. 2. The population evolution of states $|1\rangle$ and state $|2\rangle$ of such a system is presented in Fig. 4
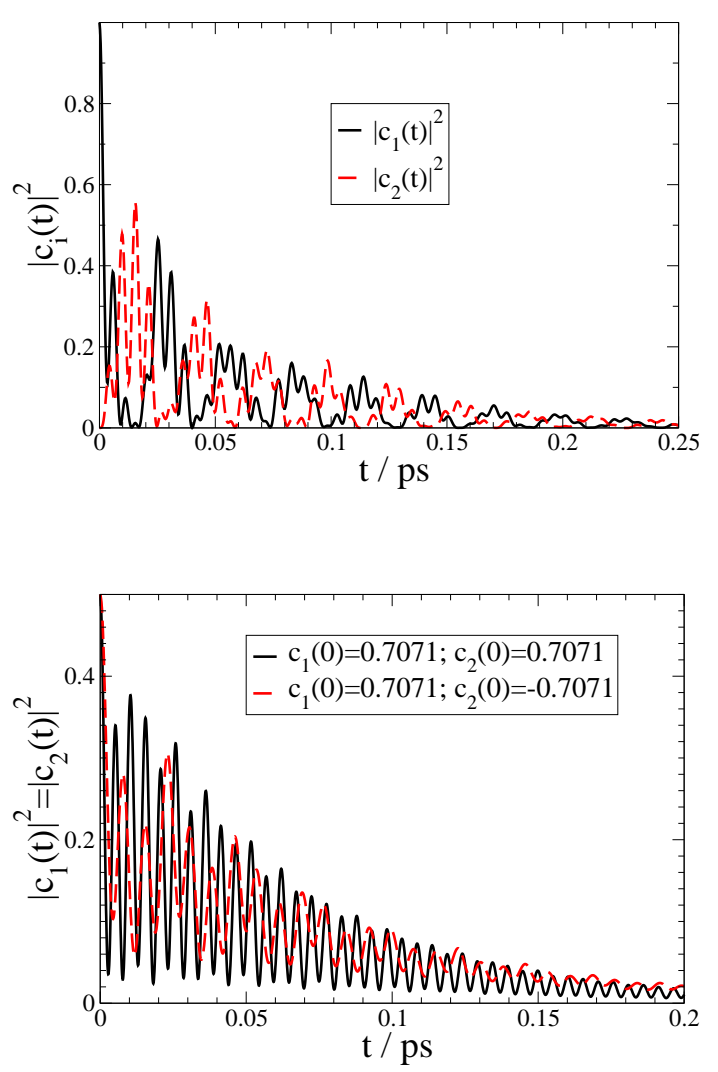

FIG. 4: (color online) Top: Population evolution of $|1\rangle$ (black solid curve) and $|2\rangle$ (red dashed curve) of a J-AGR with $\omega_{0}=$ $3.84 \mathrm{eV}$ at $D=2 \mathrm{~nm}$. Bottom: Population evolution of $|1\rangle$ of the J-AGR with $\omega_{0}=3.84 \mathrm{eV}$ at $D=2 \mathrm{~nm}$ for the initial states, $|\Psi(0)\rangle=\sqrt{0.5}|1\rangle+\sqrt{0.5}|2\rangle$ (black solid curve) and $|\Psi(0)\rangle=\sqrt{0.5}|1\rangle-\sqrt{0.5}|2\rangle$ (red dashed curve).

In the top panel of Fig. 4 the evolution of $\left|c_{1}(t)\right|^{2}$ (black solid curve) and $\left|c_{2}(t)\right|^{2}$ (red dashed curve) for initial state $|\Psi(0)\rangle=|1\rangle$ are shown. We find that both $\left|c_{1}(t)\right|^{2}$ and $\left|c_{2}(t)\right|^{2}$ decay totally within 200-250 fs. In the meanwhile, though, there is partial oscillatory exchange of population between state $|1\rangle$ and state $|2\rangle$ via the modified EM modes as indicated by the phase difference of $\pi$. Moreover, the evolution of $\left|c_{1}(t)\right|^{2}$ and $\left|c_{2}(t)\right|^{2}$ point to a rather strong non-Markovian character of the underlying dynamics, as there are very distinct oscillations on the top of the overall gradual decay of the population of these states. The population evolution of $\left|c_{1}(t)\right|^{2}$ and $\left.c_{2}(t)\right|^{2}$ for a SIS (black solid curve) and an AIS (red dashed curve) are shown in the bottom panel of Fig. 4 . We observe that, in both cases, the population of state $|1\rangle$ and $|2\rangle$ decay totally in the modified EM mode continuum within about 200 fs. Furthermore, in both cases, the overall population decay occurs with strong, gradually decreasing in magnitude, oscillations of population 
transfer between states $|1\rangle$ and $|2\rangle$ and the modified EM continuum simultaneously. However, the density of oscillations within a given time interval is lower in case of a AIS than in case of a SIS, which points to destructive quantum interference effects in the underlying strong non-Markovian population dynamics in case of an AIS. We note that a closer look at the population evolution up to $5 \mathrm{fs}$ in both panels of Fig. 4 (not shown here) shows that the decay time in each case is close to the corresponding $\tau_{e f f}^{r a d}$, instead of $\tau_{\text {eff }}$, which indicates, that the radial part of spectral density is dominating the underlying non-Markovian population dynamics here, as in the case of a QD with the same $\omega_{0}$ shown Fig. 3 .
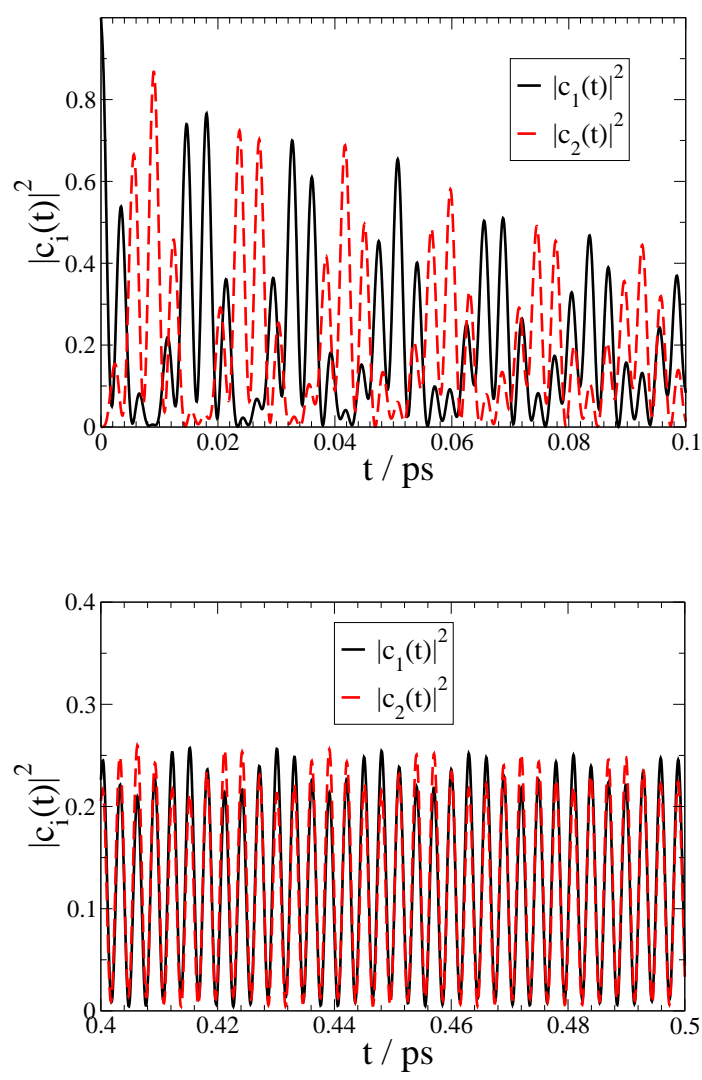

FIG. 5: (color online) Top: Population evolution of $|1\rangle$ (black solid curve) and $|2\rangle$ (red dashed curve) of a J-AGR with $\omega_{0}=$ $3.84 \mathrm{eV}$ at $D=1.55 \mathrm{~nm}$ up to $100 \mathrm{fs}$. Bottom: Population evolution of $|1\rangle$ (black solid curve) and $|2\rangle$ (red dashed curve) of a J-AGR with $\omega_{0}=3.84 \mathrm{eV}$ at $D=1.55 \mathrm{~nm}$, at later times (400-500 fs).

In Fig. 5, we study the population evolution of states $|1\rangle$ and $|2\rangle$ for a J-AGR with $\omega_{0}=3.84 \mathrm{eV}$ at $D=1.55$ $\mathrm{nm}$, with $\tau_{\text {eff }} \approx 2.3 \mathrm{fs}\left(\tau_{\text {eff }}^{\text {rad }} \approx 0.9 \mathrm{fs}\right.$ and $\left.\tau_{\text {eff }}^{\text {tan }} \approx 3.8 \mathrm{fs}\right)$, taking into account that $\lambda_{\perp}(3.84,1.55) \approx 7.50 \cdot 10^{4}$ and $\lambda_{\|}(3.84,1.55) \approx 1.85 \cdot 10^{4}$ as shown in Fig. 2. In the top panel of Fig. [5 the time evolution of $\left|c_{1}(t)\right|^{2}$ (black solid curve) and $\left|c_{2}(t)\right|^{2}$ (red dashed curve) with initial state $|\Psi(0)\rangle=|1\rangle$ are shown during the first 100 fs. At the very early stage of the population evolution of state $|1\rangle$ (not shown here), the dynamics is practically a fast decay with $\tau_{\text {eff }}$; however, very quickly the strongly non-Markovian character of the underlying dynamics manifests itself as an oscillatory transfer of population between the states $|1\rangle$ and $|2\rangle$, with the modified EM mode continuum as an intermediate, as clearly indicated by the $\pi$-phase difference between $\left|c_{1}(t)\right|^{2}$ and $\mid c_{2}(t)^{2}$. The initial population of the $\mathrm{V}$-type system is decreased to about half within the first $100 \mathrm{fs}$.
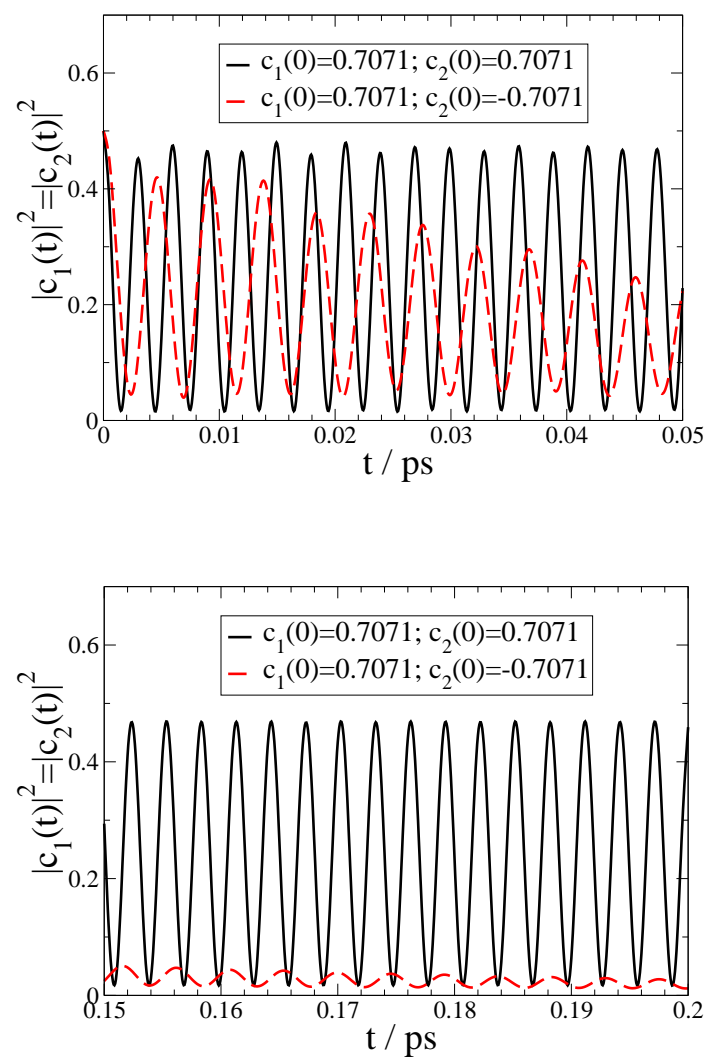

FIG. 6: (color online) Top: Population evolution of $|1\rangle$ of a JAGR with $\omega_{0}=3.84 \mathrm{eV}$ at $D=1.55 \mathrm{~nm}$ for the initial states, $|\Psi(0)\rangle=\sqrt{0.5}|1\rangle+\sqrt{0.5}|2\rangle$ (black solid curve) and $|\Psi(0)\rangle=$ $\sqrt{0.5}|1\rangle-\sqrt{0.5}|2\rangle$ (red dashed curve) up to 50 fs. Bottom: Population evolution of $|1\rangle$ of a J-AGR with $\omega_{0}=3.84 \mathrm{eV}$ at $D=1.55 \mathrm{~nm}$ for the initial states, $|\Psi(0)\rangle=\sqrt{0.5}|1\rangle+\sqrt{0.5}|2\rangle$ (black solid curve) and $|\Psi(0)\rangle=\sqrt{0.5}|1\rangle-\sqrt{0.5}|2\rangle$ (red dashed curve) at later times (150-200 fs).

In the bottom panel of Fig. 5 we present the population evolution $\left|c_{1}(t)\right|^{2}$ (black solid curve) and $\left|c_{2}(t)\right|^{2}$ (red dashed curve) at later times, in the time interval $400-500$ fs. We now find that the time-evolution of the population has reached a steady state, indicating a population exchange between the $\mathrm{V}$-type system and the 
modified EM mode continuum, which amounts in total to $\approx 50 \%$ of the initial population. Interestingly, the $\pi$ phase shift between $\left|c_{1}(t)\right|^{2}$ and $\left|c_{2}(t)\right|^{2}$, which existed at earlier times of the dynamics, shown in the top panel of this figure, is now lost. The population exchange now has now become practically a transfer of population between the states of the V-type system and the modified EM continuum, which occurs simultaneously for both states.

In Fig. 6, we investigate the influence of the initial state on the population evolution of states $|1\rangle$ and $|2\rangle$ for a J-AGR with $\omega_{0}=3.84 \mathrm{eV}$ at $D=1.55 \mathrm{~nm}$. In the top panel of this figure, we present the time evolution of $\left|c_{1}(t)\right|^{2}$ and $\left|c_{2}(t)\right|^{2}$ for a SIS (black solid curve) and an AIS (red dashed curve) for the first $50 \mathrm{fs}$. We find that the population evolution of states $|1\rangle$ and $|2\rangle$ are strongly dependent on the initial state, which indicates significant quantum interference effects in the underlying non-Markovian dynamics. In case of a SIS, the $\left|c_{1}(t)\right|^{2}$ $\left(=\left|c_{2}(t)\right|^{2}\right)$ evolution shows a steady oscillatory exchange of population between the $\mathrm{V}$-type system and the modified EM mode continuum. In case of an AIS, the situation is strikingly different; the $\left|c_{1}(t)\right|^{2}$ evolution indicates an oscillatory gradually decreasing in magnitude transfer of the initial population of the $\mathrm{V}$-type system into the modified EM mode continuum. At later times, as shown in the bottom panel of Fig. 6, a marginal, only about $5 \%$ of the initial population in total, oscillatory population transfer between states $|1\rangle$ and $|2\rangle$ and the modified EM modes, is still observable.

\section{QD dynamics at $\omega_{0}=4.16 \mathrm{eV}$}

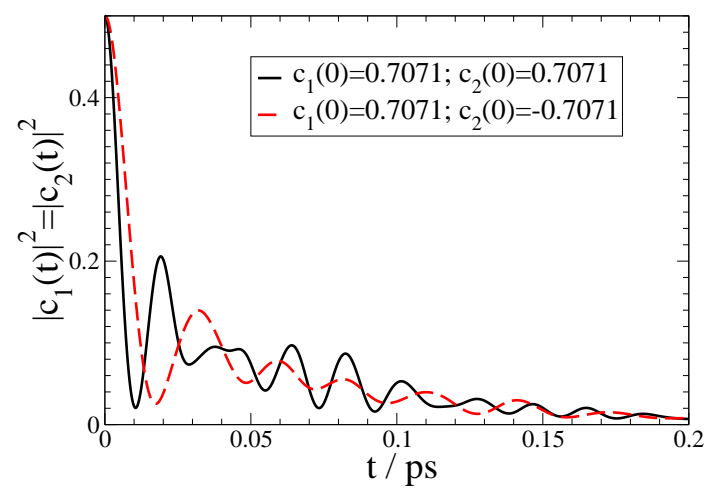

FIG. 7: (color online) Population evolution of $|1\rangle$ and $|2\rangle$ of a QD with $\omega_{0}=4.16 \mathrm{eV}$ at $D=1 \mathrm{~nm}$ for the initial states, $|\Psi(0)\rangle=\sqrt{0.5}|1\rangle+\sqrt{0.5}|2\rangle$ (black solid curve) and $|\Psi(0)\rangle=\sqrt{0.5}|1\rangle-\sqrt{0.5}|2\rangle$ (red dashed curve).

We now study the population evolution for a QD with $\omega_{0}=4.16 \mathrm{eV}$ at $D=1 \mathrm{~nm}$, with $\tau_{\text {eff }} \approx 10 \mathrm{fs}$ $\left(\tau_{e f f}^{\text {rad }} \approx 6\right.$ fs and $\left.\tau_{\text {eff }}^{\text {tan }} \approx 14 \mathrm{fs}\right)$, taking into account that $\lambda_{\perp}(4.16,1) \approx 6.54 \cdot 10^{5}$ and $\lambda_{\|}(3.84,1) \approx 2.83 \cdot 10^{5}$, as shown in Fig. 2]. In Fig. [7, we present the time evolution of $\left|c_{1}(t)\right|^{2}\left(=\left|c_{2}(t)\right|^{2}\right.$ here) in case of a SIS (black solid curve) and an AIS (red dashed curve). We observe that the population evolution is different for the two initial states, which again points to quantum interference effects in the underlying dynamics. As in case of a QD with $\omega_{0}=3.84 \mathrm{eV}$, shown in Fig. 3. the population dynamics shows a moderate non-Markovian character, and, at early times, is practically a decay with $\tau_{\text {eff }}$, while after $\approx 200 \mathrm{fs}$, the population of the upper states is (almost) completely lost. We note that the population dynamics of $|1\rangle$ and $|2\rangle$ in case of the initial state $|\Psi(0)\rangle=|1\rangle$ (not shown here) looks analogous to the corresponding dynamics in case of a $\mathrm{QD}$ with $\omega_{0}=3.84 \mathrm{eV}$ at $D=1$ $\mathrm{nm}$, presented in the top panel of Fig. 3 above.

\section{J-AGR dynamics at $\omega_{0}=4.16 \mathrm{eV}$}

We now study the population dynamics of a J-AGR, i.e. a $\mathrm{QE}$ with $\tau_{0}=70 \mathrm{ps}$, with $\omega_{0}=4.16 \mathrm{eV}$ at $D=1.55$ $\mathrm{nm}$, with $\tau_{\text {eff }} \approx 0.35 \mathrm{fs}\left(\tau_{\text {eff }}^{\text {rad }} \approx 0.2 \mathrm{fs}\right.$ and $\tau_{\text {eff }}^{\text {tan }} \approx 0.5$ fs), taking into account that $\lambda_{\perp}(4.16,1) \approx 3.21 \cdot 10^{5}$ and $\lambda_{\|}(4.16,1.55) \approx 1.39 \cdot 10^{5}$, as shown in Fig. 2. Here, the averaged effective decay rate, as well as the radial and tangential effective rates, are in attosecond time range, which is much smaller than the duration of the dynamics observed; we thus conclude that they are practically of minor importance for understanding the underlying strongly non-Markovian dynamics in this case.

In the top panel of Fig. 8, the time evolution of $\left|c_{1}(t)\right|^{2}$ (black solid curve) and $\left|c_{2}(t)\right|^{2}$ (red dashed curve) are shown for the initial state $|\Psi(0)\rangle=|1\rangle$ during the first 150 fs. The strongly non-Markovian character of the underlying dynamics manifests itself as an oscillatory exchange of population between states $|1\rangle$ and $|2\rangle$ with the modified EM mode continuum as an intermediate, as clearly indicated by the phase difference of $\pi$ between $\left|c_{1}(t)\right|^{2}$ and $\mid c_{2}(t)^{2}$. Furthermore, the initial population of the $\mathrm{V}$-type system is decreased to about $40 \%$ of its initial value within the first 150 fs.

In the bottom panel of Fig. 8 we show $\left|c_{1}(t)\right|^{2}$ (black solid curve) and $\left|c_{2}(t)\right|^{2}$ (red dashed curve) at later times, 400 - 500 fs. We now observe that the population evolution has reached a steady state; now, the non-decaying exchange of population between the $\mathrm{V}$-type system and the modified EM mode continuum amounts in total to $\approx$ $25 \%$ of the initial population. Interestingly, contrary to the findings when studying the analogous configuration at $\omega_{0}=3.84 \mathrm{eV}$ shown in Fig. [5] the $\pi$-phase difference between $\left|c_{1}(t)\right|^{2}$ and $\left|c_{2}(t)\right|^{2}$, which existed at ear- 

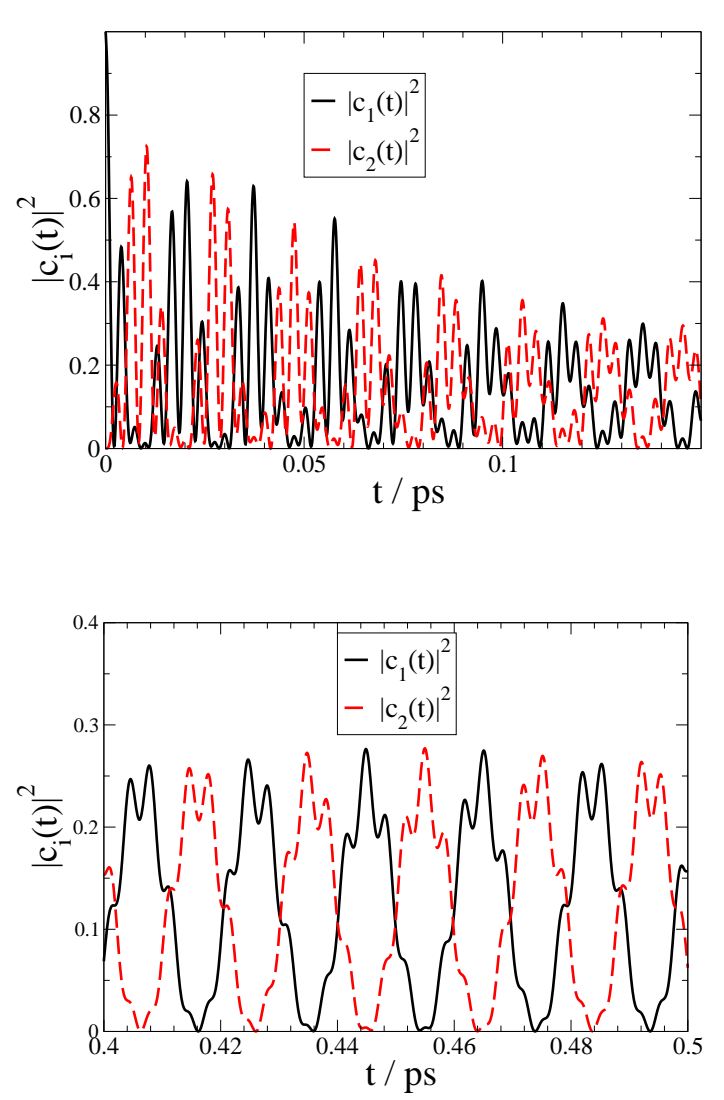

FIG. 8: (color online) Top: Population evolution of $|1\rangle$ (black solid curve) and $|2\rangle$ (red dashed curve) of a J-AGR with $\omega_{0}=$ $4.16 \mathrm{eV}$ at $D=1.55 \mathrm{~nm}$ up to 100 fs. Bottom: Population evolution of $|1\rangle$ (black solid curve) and $|2\rangle$ (red dashed curve) of a J-AGR with $\omega_{0}=4.16 \mathrm{eV}$ at $D=1.55 \mathrm{~nm}$, at later times (400-500 fs).

lier times of the dynamics, is still present; here, clearly the population exchange between the two states of the V-type system with the modified EM continuum as an intermediate, suffers no dephasing, even over long time periods, in this case.

In Fig. 9, we investigate the influence of the initial state on the population evolution of states $|1\rangle$ and $|2\rangle$ for a J-AGR with $\omega_{0}=4.16 \mathrm{eV}$ at $D=1.55 \mathrm{~nm}$. In the top panel of this figure, we present the time evolution of $\left|c_{1}(t)\right|^{2}$ and $\left|c_{2}(t)\right|^{2}$ for a SIS (black solid curve) and an AIS (red dashed curve) for the first $50 \mathrm{fs}$. We find that the population evolution of states $|1\rangle$ and $|2\rangle$ are strongly dependent on the initial state, which indicates strong quantum interference effects in the underlying non-Markovian dynamics. In case of a SIS, the $\left|c_{1}(t)\right|^{2}\left(=\left|c_{2}(t)\right|^{2}\right.$ here $)$ evolution shows a slowly decreasing in magnitude oscillatory transfer of population between the V-type system and the modified EM mode continuum. In case of an AIS, the situation is similar, although the rate of magnitude decrease is faster. At later times, as shown in the bottom panel of Fig. 9, a mod-
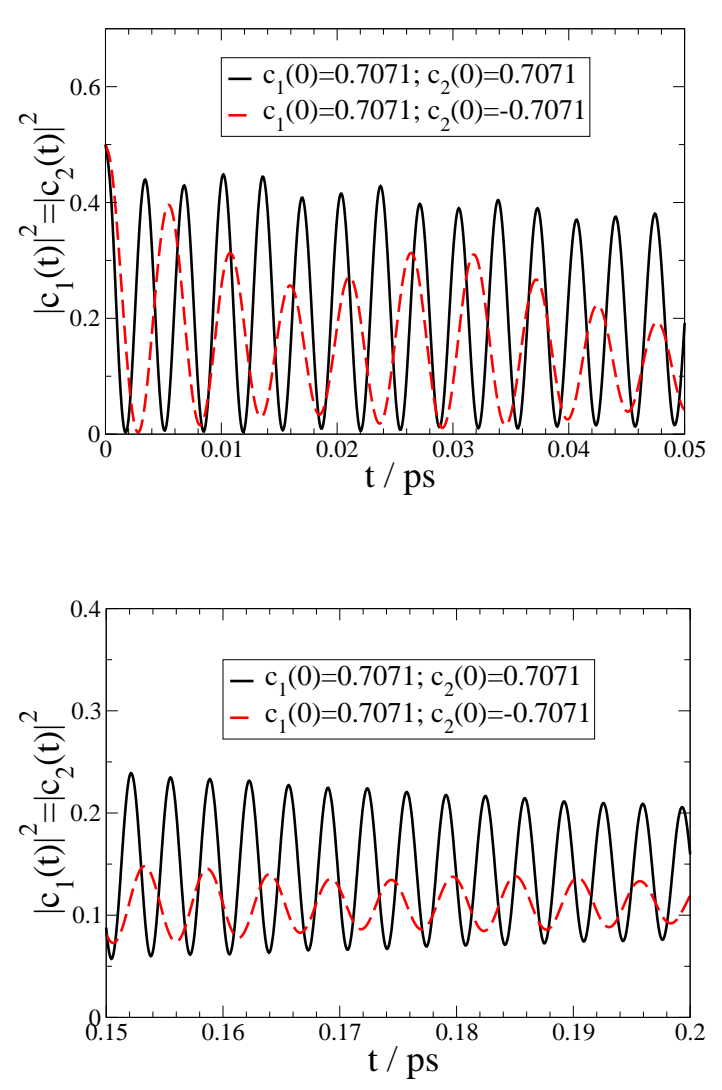

FIG. 9: (color online) Top: Population evolution of $|1\rangle$ of the J-AGR with $\omega_{0}=4.16 \mathrm{eV}$ at $D=1.55 \mathrm{~nm}$ for the initial states, $|\Psi(0)\rangle=\sqrt{0.5}|1\rangle+\sqrt{0.5}|2\rangle$ (black solid curve) and $|\Psi(0)\rangle=\sqrt{0.5}|1\rangle-\sqrt{0.5}|2\rangle$ (red dashed curve) up to 50 fs. Bottom: Population evolution of $|1\rangle$ of the JAGR with $\omega_{0}=4.16 \mathrm{eV}$ at $D=1.55 \mathrm{~nm}$ for the initial states, $|\Psi(0)\rangle=\sqrt{0.5}|1\rangle+\sqrt{0.5}|2\rangle$ (black solid curve) and $|\Psi(0)\rangle=\sqrt{0.5}|1\rangle-\sqrt{0.5}|2\rangle$ (red dashed curve) at later times (150-200 fs).

erate oscillatory population exchange, of about $20 \%$ and $10 \%$ of the initial population, in case of a SIS and an AIS, respectively, between states $|1\rangle$ and $|2\rangle$ and the modified EM modes occurs simultaneously. These minor qualitative differences on the population evolution of $|1\rangle$ and $|2\rangle$ are safely attributed to quantum interference effects in the underlying non-Markovian dynamics. Most interestingly, however, for both initial states, at later times, the population evolution shows clearly that about $20 \%$ of the initial population is "trapped" in the V-type system at all times, which indicates that coherent population trapping 38 under conditions of strong coupling between a QE and a MNP, as studied here, is observable. 


\section{E. The influence of the FCA on the dynamics of the V-type QE: $K^{ \pm}(\tau)$ vs $K_{F C A}^{ \pm}(\tau)$}

We now investigate the influence of the FCA on the non-Markovian spontaneous emission dynamics of a $\mathrm{V}$ type QE. In the top panel of Fig. 10, we present the population evolution of state $|1\rangle$ with initial state $|\Psi(0)\rangle=$ $|1\rangle$, for a J-AGR at $D=1.55 \mathrm{~nm}$ with $\omega_{0}=3.84 \mathrm{eV}$, obtained using the $K^{ \pm}(\tau)$ kernel (black solid curve), defined by Eq. (15), and using the $K_{F C A}^{ \pm}(\tau)$ kernel (red dashed curve), defined by Eq. (17). We observe that the FCA introduces a phase shift between the two $\left|c_{1}(t)\right|^{2}$ curves. More specifically, the $\left|c_{1}(t)\right|^{2}$ obtained with $K_{F C A}^{ \pm}(\tau)$ precedes the corresponding population evolution when the FCA is not invoked; we denote such a phase shift between the two curves as positive. In the bottom panel of Fig. 10, we present the population dynamics of state $|1\rangle$ for a J-AGR, again at $D=1.55 \mathrm{~nm}$, but now with $\omega_{0}=4.16 \mathrm{eV}$, obtained using the $K^{ \pm}(\tau)$ kernel (black solid curve) and with the $K_{F C A}^{ \pm}(\tau)$ kernel (red dashed curve). We once more find that the two $\left|c_{1}(t)\right|^{2}$ curves are phase-shifted to each other. However, interestingly, in this case, the phase shift is negative.

In order to better understand the phase shift in the population dynamics introduced by invoking the FCA, as discussed above, we further focus on the radial spectral density $J^{r a d}(\omega)$ and $J_{F C A}^{r a d}(\omega)$, with and without the FCA, respectively, to be used in the corresponding kernels. We do not elaborate on the case of the tangential spectral density here, since one comes to analogous findings as for the radial spectral case, to be presented now.

In Fig. 11] we show the $J^{r a d}(\omega)$ (black solid curve) and $J_{F C A}^{r a d}(\omega)$ (red dashed curve) in case of a QE with $\omega_{0}=3.84 \mathrm{eV}$ (top panel) and $\omega_{0}=4.16 \mathrm{eV}$ (bottom panel). We observe that in both cases, around the resonance frequency $\omega_{0}, J^{r a d}(\omega)$ and $J_{F C A}^{r a d}(\omega)$ are practically the same, which implies that in the common case of a (radial) spectral density dominated by only one plasmonic resonance, or more generally, by several non-overlapping resonances, calculating the non-Markovian dynamics of a QE in the proximity of a MNP, with or without the FCA, leads to practically indistinguishable results. However, when the (radial) spectral density is dominated by overlapping plasmonic resonances, as in this work (see Fig. 2), invoking the FCA leads to observable differences between the two spectral densities as one moves away from the resonance frequency of the QE. In particular, in presence of overlapping resonances in the spectral density, $J_{F C A}^{r a d}(\omega)<J^{r a d}(\omega)$ is valid when $\omega>\omega_{0}$, with reversed relation between the two spectral densities, when $\omega<\omega_{0}$. Furthermore, with help of Fig. 11, we can rationalise qualitatively the phase difference in the evolution of $\left|c_{1}(t)\right|^{2}$, when obtained with $K_{F C A}^{ \pm}(\tau)$, instead of $K^{ \pm}(\tau)$. We conclude that the introduction of the FCA for computing the population dynamics of a $\mathrm{QE}$ near
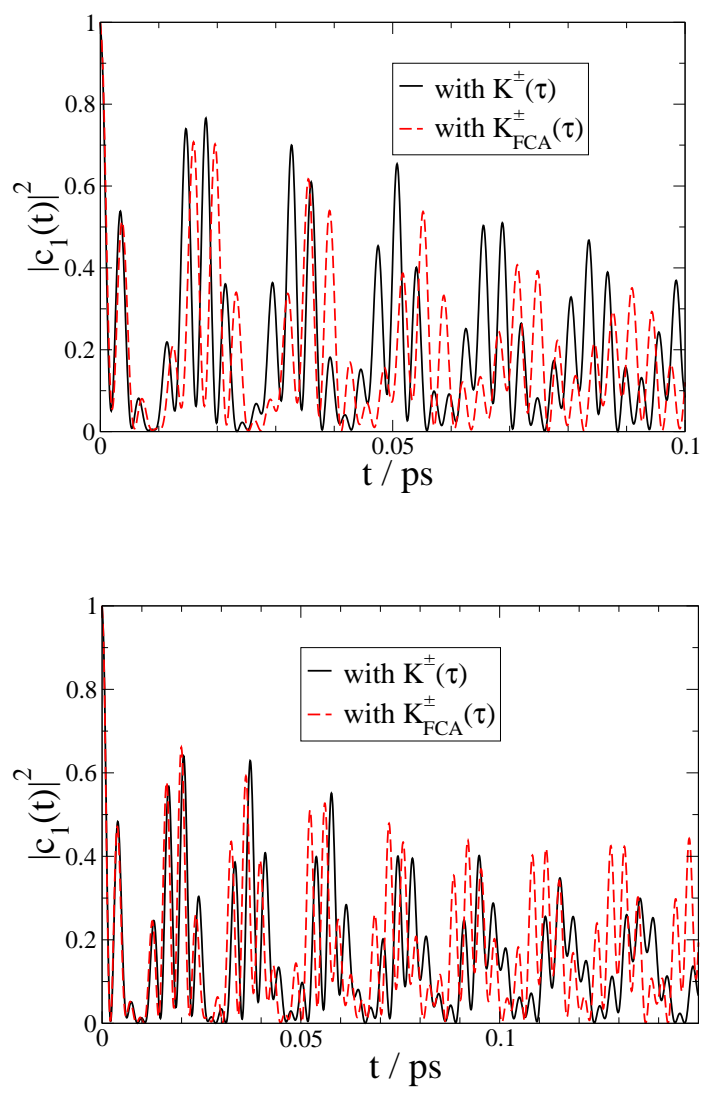

FIG. 10: (color online) Population evolution of $|1\rangle$ of a QE with $\tau_{0}=70 \mathrm{ps}$ at $D=1.55 \mathrm{~nm}$ obtained using the kernel $K^{ \pm}(\tau)$ (defined by Eq. (15)) (black solid curve) and using the kernel $K_{F C A}^{ \pm}(\tau)$ (defined by Eq. (17)) (red dashed curve), with $\omega_{0}=3.84 \mathrm{eV}$ (top) and $\omega_{0}=4.16 \mathrm{eV}$ (bottom). See text for discussion.

a MNP, for which the spectral density is dominated by overlapping plasmonic resonances, introduces a positive (negative) phase shift in the population evolution, when the $\mathrm{QE}$ resonance frequency lies in the lower (higher) end of its non-vanishing spectral density.

\section{DYNAMICS OF A TWO-LEVEL QE NEAR A MNP}

In this section, we consider a V-type QE, for which, however, the transition dipole moments $\vec{\mu}_{10}$ and $\vec{\mu}_{20}$ are along the $z$ and the $x$ axis, i.e. we use $\vec{\mu}_{10}=\mu \hat{\epsilon}_{z(x)}$ and $\vec{\mu}_{20}=\mu \hat{\epsilon}_{x(z)}$ in Eq. (7). In such a case, since $K^{i j}(\tau)=0$ $(i \neq j)$, the population dynamics of one excited state of the $\mathrm{V}$-type system does not depend on the dynamics of the other excited state; therefore one has effectively two independent two-level systems, instead of a three-level 

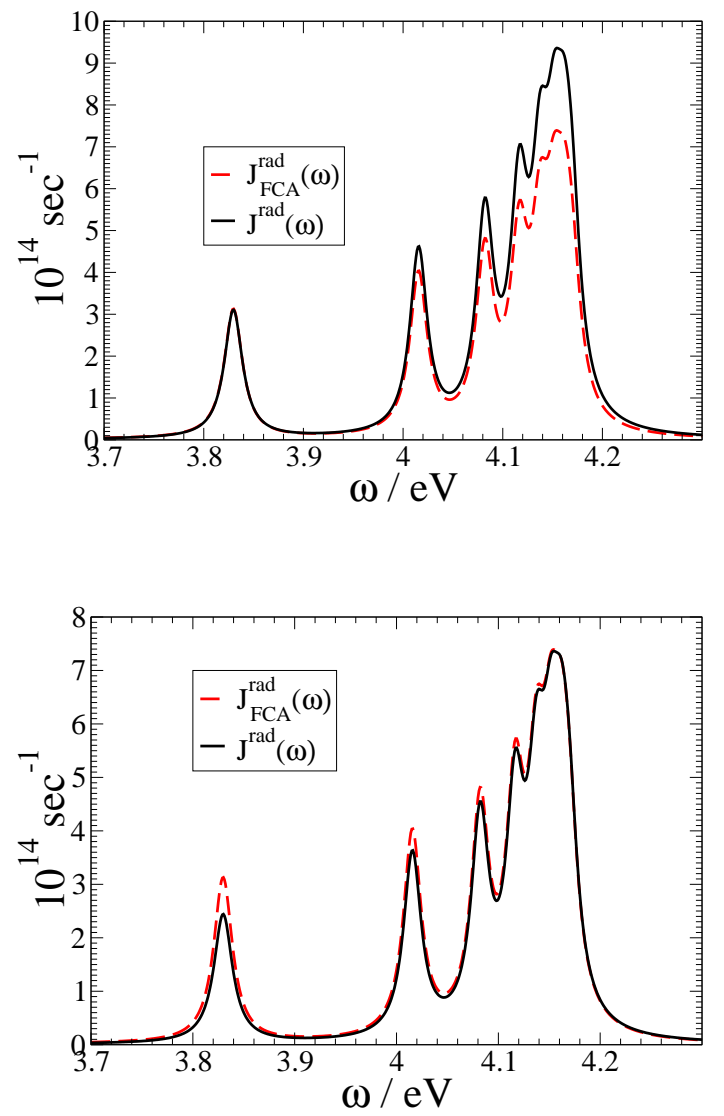

FIG. 11: (color online) The radial spectral density $J^{\text {rad }}(\omega)$ (black solid curve) and $J_{F C A}^{r a d}(\omega)$ (red dashed curve) for a QE with $\tau_{0}=70 \mathrm{ps}$ at $D=1.55 \mathrm{~nm}$ with $\omega_{0}=3.84 \mathrm{eV}$ (top) and $\omega_{0}=4.16 \mathrm{eV}$ (bottom). See text for discussion.

V-type system. We also note that the two-level transition dipole polarisation $\vec{\mu}$ along the $z$ (along the $x$ axis) implies that the population dynamics of such a two-level system is affected by the radial (tangential) spectral density only.

We now study the dynamics for such a two-level system in order to compare its population dynamics to the dynamics of a $V$-type system presented above. For the sake of concreteness, we focus on a two-level QE, composed of states $|0\rangle$ and $|1\rangle$ (see Fig. 1), with resonance frequency $\omega_{0}=4.16 \mathrm{eV}$; the results at a lower $\omega_{0}$ (not shown here) are similar to the results at $\omega_{0}=4.16 \mathrm{eV}$, with only minor quantitative differences due to the much smaller enhancement factors $\lambda^{\perp}\left(\omega_{0}, D\right)$ and $\lambda^{\|}\left(\omega_{0}, D\right)$, when $\omega_{0}<4.16 \mathrm{eV}$.

In Fig. 12, we present the population dynamics of state $|1\rangle$ of a two-state QE with $\tau_{0}=4$ ns at $D=1 \mathrm{~nm}$, with a transition dipole moment along the $z$ axis (black solid curve) and along the $x$ axis (red dashed curve); the corresponding effective decay times $\tau_{\text {eff }}^{\text {rad }}$ and $\tau_{\text {eff }}^{\text {tan }}$

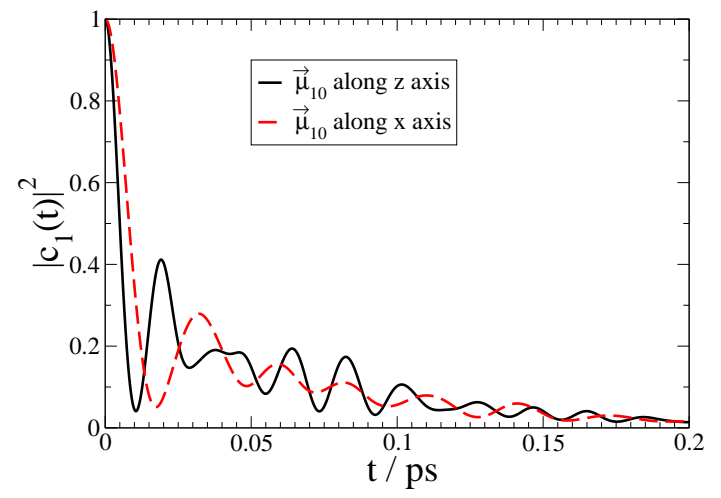

FIG. 12: (color online) Population evolution of $|1\rangle$ of a twolevel system with $\omega_{0}=4.16 \mathrm{eV}$ and $\tau_{0}=4 \mathrm{~ns}$ at $D=1 \mathrm{~nm}$ with $\vec{\mu}_{10}=\mu \hat{\epsilon}_{z}$ (black solid curve) and $\vec{\mu}_{10}=\mu \hat{\epsilon}_{x}$ (red dashed curve). See text for discussion.

are $6 \mathrm{fs}$ and $14 \mathrm{fs}$, respectively, taking into account that $\lambda^{\perp}(4.16,1) \approx 6.54 \cdot 10^{5}$ and $\lambda^{\|}(3.84,1) \approx 2.83 \cdot 10^{5}$ as shown in Fig. 2. The time evolution of $\left|c_{1}(t)\right|^{2}$ at early times can be understood as a decay with the corresponding effective decay time. However, quickly, an oscillatory decay of the population of $|1\rangle$ into the modified EM mode continuum with distinctly non-Markovian character dominates the dynamics. Moreover, an interesting conjecture regarding the population dynamics shown in this figure can be made, when a comparison with the population dynamics for the $V$-type system with $\omega_{0}=4.16 \mathrm{eV}$ and $\tau_{0}=4 \mathrm{~ns}$ at $D=1 \mathrm{~nm}$ presented in Fig. 7 above is made. More specifically, we observe that the curves of $\left|c_{1}(t)\right|^{2}$ in Fig. 7 and in Fig. 12 look very similar, when taking into account the different amount of initial population, although they correspond to distinctly different initial conditions. The comparison of the results of these two figures suggests strongly that the different population evolution of $\left|c_{1}(t)\right|^{2}$ for the SIS and the AIS shown in Fig. 7 is due to the fact that for a SIS state, due to quantum interference, the dynamics is dominated by the spectral density along the $z$ axis, i.e. the radial spectral density. In case of an AIS, the $\left|c_{1}(t)\right|^{2}$ evolution in Fig. 7 is dominated by the tangential spectral density, i.e. along the $x$ axis ${ }^{31}$. We can thus understand better, with help of the two-level system studied here, the direct result of the quantum interference on the dynamics of the $\mathrm{V}$-type system with $\omega_{0}=4.16 \mathrm{eV}$ and $\tau_{0}=4 \mathrm{~ns}$ at $D=1 \mathrm{~nm}$, discussed above.

In Fig. 13, we consider a two-level QE with $\omega_{0}=4.16$ $\mathrm{eV}$ and $\tau_{0}=70 \mathrm{ps}$ with a transition dipole moment along the $z$ axis, which implies that the dynamics is affected only by the radial spectral density. In this figure we present the population evolution $\left|c_{1}(t)\right|^{2}$ at $D=1.85 \mathrm{~nm}$ (black solid curve) and at $D=2 \mathrm{~nm}$ (red dashed curve) 

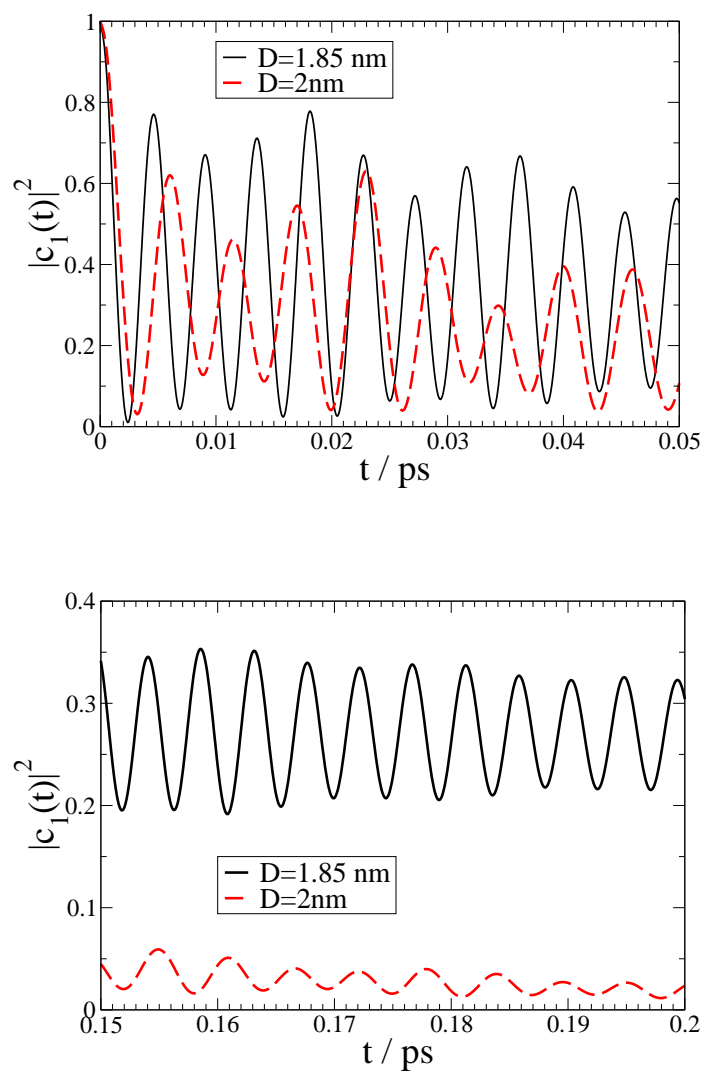

FIG. 13: (color online) Population evolution of $|1\rangle$ of a twolevel system with $\tau_{0}=70 \mathrm{ps}$ and transition dipole moment along the $z$ axis at $D=1.85 \mathrm{~nm}$ (black solid curve) and at $D=2 \mathrm{~nm}$ (red dashed curve) up to $100 \mathrm{fs}$ (top panel), and at later times, 200-300 fs (bottom panel). See text for discussion.

up to 50 fs (top panel) and at later times, 150-200 fs (bottom panel). Here, the radial effective decay times $\tau_{\text {eff }}^{r a d}$ and $\tau_{\text {eff }}^{t a n}$ are about $0.5 \mathrm{fs}$ and $1.5 \mathrm{fs}$, respectively, taking into account that $\lambda^{\perp}(4.16,1.85) \approx 1.39 \cdot 10^{5}$ and $\lambda^{\perp}(4.16,2) \approx 4.86 \cdot 10^{4}$ as shown in Fig. 2 .

In the top panel of Fig. 13, the population evolution of state $|1\rangle$ at $D=1.85 \mathrm{~nm}$ and at $D=2 \mathrm{~nm}$ shows strong non-Markovian character; only at very early times, the decay dynamics is dictated by the corresponding $\tau_{e f f}^{r a d}$. After $50 \mathrm{fs}$, at $D=1.85 \mathrm{~nm}$, the population of state $|1\rangle$ is decreased to about half its initial value, which resembles the time evolution of $\left|c_{1}(t)\right|^{2}$ with an AIS of a V-type system with resonance frequency $4.16 \mathrm{eV}$ and free-space decay time $70 \mathrm{ps}$ at $D=1.55 \mathrm{~nm}$, shown in Fig. 9. We thus conclude that the coupling conditions of the twolevel QE here are comparable to the coupling conditions of the V-type QE related to the results shown in Fig. 9. a comparative discussion of the population dynamics shown in these two figures is then justifiable. With help of the results of the two-level system shown in Fig. 13. we can now conclude that the evolution of $\left|c_{1}(t)\right|^{2}$ of a V-type system with an AIS, as shown in the top panel of Fig. 9 is dominated primarily by the radial spectral density of the modified EM modes.

In the bottom panel of Fig. 13, we find that at $D=1.85 \mathrm{~nm}$, at later times, there is a steady population exchange of about $15 \%$ of the initial population between state $|1\rangle$ and the modified radial EM modes, while at $D=2 \mathrm{~nm}$, the corresponding population exchange amounts only to about $5 \%$. Most importantly, at $D=1.85 \mathrm{~nm}$, the population exchange occurs while about $20 \%$ of the initial population remains in the twolevel $\mathrm{QE}$ at all times. At $D=2 \mathrm{~nm}$, however, the initial population of state $|1\rangle$ is almost completely transferred in the EM continuum, with clear indication that it will ultimately wane out totally. We thus conclude that population trapping is also observable for a two-level QE near a MNP under the given conditions.

We have also studied the influence of the FCA in the dynamics of a two-level system (not shown here); our conclusions are similar to the case of a $\mathrm{V}$-type system discussed above.

\section{CONCLUSIONS}

In conclusion, we have studied the non-Markovian quantum dynamics for various initial states of a degenerate V-type QE near a MNP, which exhibits anisotropic Purcell effect. We have considered QEs with free-space decay time in the ns and ps time regime, for various resonance frequencies, corresponding to moderate $\left(10^{4}\right)$ and strong $\left(10^{5}\right)$ enhancement of the free-space decay rate. We observe a transition in the upper states population time evolution, from a gradual decay of the total population to a steady oscillatory population exchange between the QE and the modified EM modes. This effect occurs when strong enhancement of the free-space spontaneous decay rate of the $\mathrm{QE}$ occurs, and, in some cases it can lead to coherent population trapping in the V-type system. Furthermore, the strong dependence of the population dynamics on a particular initial state, at otherwise identical conditions, clearly indicates that spontaneous emission interference of the $\mathrm{QE}$ excited states into the modified EM modes continuum takes place. We have also studied a two-level QE near a MNP at the same conditions as the $\mathrm{V}$-type system, coming to similar conclusions as for the three-level system.

Lastly, we have investigated the influence of the FCA on the calculated population dynamics for the above three- and two-level systems. In both cases, we conclude that the FCA can affect the quality of the calculated population dynamics only in systems, for which the modified by the MNP spectral density of the EM modes is dominated by overlapping plasmonic resonances. In such cases, the FCA introduces a positive (negative) phase shift in the population time evolution with respect to the exact dynamics without invoking the FCA; a positive (negative) phase shift appears when the resonance frequency of the QE lies in the low (high) end of the spec- 
tral density. In case of spectral densities with only nonoverlapping plasmonic resonances, however, the FCA is an excellent approximation. We believe that our findings can be particularly useful in the development of quantum technological applications involving plasmonic nanostructures 39 .

\section{Acknowledgments}

E.P. acknowledges the support of "Research Projects for Excellence IKY/Siemens".
* Electronic address: ithano@eie.gr

† Electronic address: vyannop@mail.ntua.gr

‡ Electronic address: paspalak@upatras.gr

1 L. Novotny and N. F. van Hulst, Nature Photonics 5, 83 (2011).

2 V. Giannini, A. I. Fernadez-Dominquez, S. C. Heck, and S. A. Maier, Chem. Rev. 111, 3888 (2011).

3 S. Schlucker, Angew. Chem. Int. Ed. 53, 4756 (2014).

${ }^{4}$ H. Atwater and A. Polman, Nature Mater. 9, 205 (2010).

${ }^{5}$ L. Piatkowski, N. Accanto, and N. F. van Hulst, ACS Photonics 3, 1401 (2016).

${ }^{6}$ F. Vollmer and L. Yang, Nanophotonics 1, 267 (2012).

7 A. Gonzalez-Tudela, F. J. Rodriguez, L. Quiroga, and C. Tedejor, Phys. Rev. B 82, 115334 (2010).

8 T. Hümmer, F. J. Garcia-Vidal, L. Martin-Moreno, and D. Zueco, Phys. Rev. B 87, 115419 (2013).

9 A. Gonzalez-Tudela, P. A. Huidobro, L. Martin-Moreno, C. Tejedor, and F. J. Garcia-Vidal, Phys. Rev. B 89, 041420(R) (2014).

10 A. Delga, J. Feist, J. Bravo-Abad, and F. J. Garcia-Vidal, Phys. Rev. Lett. 112, 253601 (2014).

11 J. Hakami, L. Wang, and M. S. Zubairy, Phys. Rev. A 89, 053835 (2014).

12 J. Hakami and M. S. Zubairy, Phys. Rev. A 93, 022320 (2016).

13 E. Dulkeith, A. C. Morteani, T. Niedereichholz, T. A. Klar, J. Feldmann, S. A. Levi, F. C. J. M. van Veggel, D. N. Reinhoudt, M. Möller, and D. I. Gittins, Phys. Rev. Lett. 89, 203002 (2002).

14 S. Kühn, U. Håkanson, L. Rogobete, and V. Sandoghar, Phys. Rev. Lett. 97, 017402 (2006).

15 P. Anger, P. Bharadwaj, and L. Novotny, Phys. Rev. Lett. 96, 113002 (2006).

16 A. Kinkhabwala, Z. Yu, S.-H. Fan, Y. Avlasevich, K. Müllen, and W. E. Moerner, Nature Photonics 3, 654 (2009).

17 A. Huck, S. Kumar, A. Shakoor, and U. L. Andersen, Phys. Rev. Lett. 106, 096801 (2011).

18 T. B. Hoang, G. M. Akselrod, C. Argyropoulos, J. Huang, D. R. Smith, and M. H. Mikkelsen, Nature Commun. 6, 7788 (2015).

19 T. B. Hoang, G. M. Akselrod, and M. H. Mikkelsen, Nano Lett. 16, 270 (2016).
20 R. Chikkaraddy, B. de Nijs, F. Benz, S.J. Barrow, O.A. Scherman, E. Rosta, A. Demetriadou, P. Fox, O. Hess, and J.J. Baumberg, Nature 535, 127 (2016).

21 R.-Q. Li, D. Hernangomez-Perez, F.J. Garcia-Vidal, and A.I. Fernandez-Dominguez, Phys. Rev. Lett. 117, 107401 (2016).

22 G. S. Agarwal, Phys. Rev. Lett. 84, 5500 (2000).

23 M. Kiffner, M. Macovei, J. Evers, and C. H. Keitel, Progress in Optics, ed. E. Wolf, Vol. 55, (2010), p. 85.

24 G. X. Li, F.-L. Li, and S.-Y. Zhu, Phys. Rev. A 64, 013819 (2001).

25 Y.-P. Yang, J.-P. Xu, H. Chen, and S.-Y. Zhu, Phys. Rev. Lett. 100, 043601 (2008).

${ }^{26}$ G.-X. Li, J. Evers, and C. H. Keitel, Phys. Rev. B 80, $045102(2009)$.

27 J.-P. Xu and Y.-P. Yang, Phys. Rev. A 81, 013816 (2010).

28 P. K. Jha, X. Ni, C. Wu, Y. Wang, and X. Zhang, Phys. Rev. Lett. 115, 025501 (2015).

29 L. Sun and C. Jiang, Opt. Express 24, 7719 (2016).

30 V. Yannopapas, E. Paspalakis, and N. V. Vitanov, Phys. Rev. Lett. 103, 063602 (2009).

31 S. Evangelou, V. Yannopapas, and E. Paspalakis, Phys. Rev. A 83, 055805 (2011); ibid 83, 023819 (2011).

${ }^{32}$ Y. Gu, L. Wang, P. Ren, J.-X. Zhang, T.-C. Zhang, O. J. F. Martin, and Q.-H. Gong, Nano Lett. 12, 2488 (2012).

33 I. Thanopulos, P. Brumer, and M. Shapiro, J. Phys. Chem. 133, 154111 (2010); X. Li, I. Thanopulos, and M. Shapiro, Phys. Rev. A 83, 033415 (2011).

34 V. Yannopapas and N. V. Vitanov, Phys. Rev. B 75, 115124 (2007).

35 H. T. Dung, L. Knöll, and D.-G. Welsch, Phys. Rev. A 62, 053804 (2000).

${ }^{36}$ A. V. Akimov, A. Mukherjee, C. L. Yu, D. E. Chang, A. S. Zibrov, P. R. Hemmer, H. Park, and M. D. Lukin, Nature (London) 450, 402 (2007).

37 H. Fidder, J. Knoester, and D. A. Wiersma, Chem. Phys. Lett. 171, 529 (1990).

38 E. Paspalakis and P. L. Knight, Phys. Rev. Lett. 81, 293 (1998).

39 J. Ren, T. Wu, and X. Zhang, Scientific Reports 5, 13941 (2015). 